\title{
Prise en charge du myélome multiple
}

\author{
Ann S. Lee MD MÉd, Roger Y. Tsang MD, Irwindeep Sandhu MD
}

Citation : CMAJ 2021 August 30;193:E1349. doi : 10.1503/cmaj.202824-f

Voir la version anglaise de l'article ici : www.cmaj.ca/lookup/doi/10.1503/cmaj.202824
1 Le myélome multiple est la deuxième hémopathie maligne la plus fréquente
1 au Canada
L'incidence du myélome multiple est de 55 cas par 1000000 de personnes au Canada et la prévalence est en croissance ${ }^{1}$. De nouveaux traitements, comme les médicaments immunomodulateurs et les inhibiteurs du protéasome, ont augmenté la survie sans progression de la maladie à 5 ans de $29 \%$ en 1997 à $68 \%$ en 2014 parmi les patients qui ont reçu une greffe de cellules souches autologues².

2

Tous les patients atteints de myélome multiple devraient être orientés de façon précoce afin d'obtenir une évaluation de leur admissibilité à la greffe

On détermine l'admissibilité au traitement, y compris à la greffe de cellules souches autologues, non seulement en fonction de l'âge et de la fonction rénale, mais aussi des comorbidités et du statut fonctionnel ${ }^{3}$. Le taux de mortalité à 100 jours après la greffe, toutes causes confondues, est semblable pour les jeunes patients ( $40-60$ ans) et les patients plus âgés ( $\geq 60$ ans) $(1 \% \text { c. } 2 \%)^{3}$.

On recommande l'administration de bisphosphonates pour tous les patients atteints de myélome multiple, que des signes de maladie osseuse soient présents ou non

L'administration d'acide zolédronique et de pamidronate prévient l'ostéopénie, les lésions lytiques et les fractures. La posologie et la fréquence dépendent des indications et des facteurs de risque ${ }^{4}$. Employer des bisphosphonates sous forme intraveineuse plus puissants augmente cependant le risque d'ostéonécrose de la mâchoire; des examens dentaires réguliers peuvent atténuer les risques qui y sont associés.

L'antibioprophylaxie, les suppléments d'immunoglobulines et les vaccins inactivés peuvent atténuer les infections

Les maladies et les facteurs liés aux traitements entraînent une augmentation du risque d'infections virales et bactériennes qui est 10 fois et 7 fois supérieure, respectivement, pour les patients atteints de myélome multiple ${ }^{5}$. Une antibioprophylaxie aux fluoroquinolones est souvent prescrite par l'équipe de spécialistes aux patients amorçant un traitement actif dans les 3 premiers mois suivant le diagnostic ${ }^{5}$. Chez les patients présentant de fréquentes infections ou des infections graves, on peut considérer l'utilisation de suppléments d'immunoglobulines, accompagnés ou non d'une antibiothérapie à long terme. On devrait administrer des vaccins inactivés contre la grippe et le pneumocoque (vaccin antipneumococcique polysaccharidique) à tous les patients.

Les cliniciens devraient employer des seuils peu élevés pour procéder

3 à des biopsies cutanées de nouvelles lésions présentant des caractéristiques préoccupantes

Les patients atteints d'un myélome multiple ont un risque accru de cancer de la peau, indépendamment de leur âge ou de leur ethnicité ${ }^{6}$. Tout particulièrement, ces patients présentent un taux de carcinome épidermoïde 2,4 fois plus important que celui des cas-témoins appariés selon l'âge. 


\section{Références}

1. Tsang M, Le M, Ghazawi FM, et al. Multiple myeloma epidemiology and patient geographic distribution in Canada: a population study. Cancer 2019;125:2435-44

2. Nishimura KK, Barlogie B, van Rhee F, et al. Long-term outcomes after autologous stem cell transplantation for multiple myeloma. Blood Adv 2020;4:422-31.

3. Mikhael J, Ismaila N, Cheung MC, et al. Treatment of multiple myeloma: ASCO and CCO joint clinical practice guideline. [erratum publié dans J Clin Oncol 2020;38:2469]. J Clin Oncol 2019;37:1228-63.

4. Anderson K, Ismaila N, Flynn PJ, et al. Role of bone-modifying agents in multiple myeloma: American Society of Clinical Oncology clinical practice guideline update. J Clin Oncol 2018;36:812-8.

5. Blimark C, Holmberg E, Mellqvist UH, et al. Multiple myeloma and infections: a population-based study on 9253 multiple myeloma patients. Haematologica 2015;100:107-13.

6. Robinson AA, Wang J, Vardanyan S, et al. Risk of skin cancer in multiple myeloma patients: a retrospective cohort study. Eur J Haematol 2016;97:439-44.

Intérêts concurrents : Irwindeep Sandhu a reçu des honoraires de Celgene/BMS, Amgen, Gilead/Kite, Janssen, Takeda, Beigene, Novartis et Pfizer pour la présentation de conférences et pour avoir siégé à des conseils; il possède aussi des actions de IllumiSonics inc. Aucun autre intérêt concurrent n'a été déclaré.

Cet article a été révisé par des pairs.

Affiliations : Département de médecine de familiale (Lee), Université de l'Alberta, Edmonton, Alb.; Département d'oncologie (Tsang), Centre de cancérologie Tom Baker, Université de Calgary, Calgary, Alb.; Département d'oncologie médicale (Sandhu), Université de l'Alberta, Institut de cancérologie Cross, Edmonton, Alb.

Propriété intellectuelle du contenu : Il s'agit d'un article en libre accès distribué conformément aux modalités de la licence Creative Commons Attribution (CC BY-NC-ND 4.0), qui permet l'utilisation, la diffusion et la reproduction de tout médium à la condition que la publication originale soit adéquatement citée, que l'utilisation se fasse à des fins non commerciales (c.-à-d., recherche ou éducation) et qu'aucune modification ni adaptation n'y soit apportée. Voir : https://creativecommons.org/licenses/by-nc-nd/4.0/deed.fr.

Remerciements : Les auteurs souhaitent remercier les $D^{\text {res }}$ Michelle Morros et Alison White pour leurs commentaires sur les versions antérieures de ce manuscrit.

Correspondance : Ann Lee, ann.lee@ualberta.ca

Le JAMC vous invite à soumettre vos textes pour la rubrique « Cinq choses à savoir ... » en ligne à http://mc. manuscriptcentral.com/cmaj. 